\title{
Chinese Rural Sports Public Service Funds Input Current Status Analysis and Prediction
}

\author{
Hongbin Cai ${ }^{1, *}$ and Ying Wang ${ }^{2}$ \\ ${ }^{I}$ Institute of Physical Education, Fuyang Teachers College, Fuyang 236000, Anhui, China; ${ }^{2}$ Attached Primary School, \\ Fuyang Teachers College, Fuyang 236000, Anhui, China
}

\begin{abstract}
In the background of Chinese sports rapidly development, it still has lots of problems and many factors that restrict Chinese sports development. Among them, rural sports are one of the main factors. The paper starts from Chinese rural sports public service funding input prediction and current status analysis two aspects, and goes ahead with comprehensive researches through trend extrapolation prediction model and statistical analysis method. Firstly, it utilizes trend extrapolation prediction's modified exponential curve approach to make quantitative analysis of Chinese rural sports public service funds input from 2007 to 2012, and then predict Chinese rural sports funds input changes in five years. Secondly, it makes use of statistical analysis to study Chinese rural sports public service funds input current status, and gets conclusions that the amplification of Chinese rural sports funds input occupied percentage in overall fiscal expenditure is not big, sports consciousness is not strong, sports facilities utilization is incomplete, sports activities places construction is imperfect, sports personnel management system is unsound, sports field and equipment maintenance is not formal, all of these are key problems that Chinese rural sports public service development should consider at present stage.
\end{abstract}

Keywords: Modified exponential curve approach, public service, rural sports, statistical analysis, trend extrapolation prediction.

\section{INTRODUCTION}

Funds shortage, organizational structure imperfectness, sports consciousness not going deeper, all are one of numerous problems that Chinese rural sports exists at present stage. Over the years, scholars have made many researches and gained achievements [1].

Yan Qin in the article "Hubei province sports public service equalization fiscal guarantee mechanism", took Hubei province as an example, studied sports service equalization and its fiscal guarantee mechanism. The thesis pointed out that Hubei province sports public service has already moved toward another stage, all aspects have improved, its fiscal guarantee mechanism have also been relative perfected, and then built good foundation for Hubei province sports development. Gao Kui-Ting in the article "Urban and rural sports public service equalization evaluation indicator system construction research", from the perspective of city sports, studied sports public service equalization problem. The thesis pointed out that urban and rural sports public service equalization system construction was the key to propel to city sports development, among urban and rural sports public service numerous indicators, each evaluation factor functions were different that should be taken into comprehensive consideration [2].

Fan Dong-Yun in the article "Guangzhou city sports public service research", by researching Guangzhou city sports status, analyzed its sports public service existed problems and corresponding solutions. The thesis pointed out: Guangzhou city sports public service should base on masses, do a good job for the masses, realistic improve masses' sports consciousness, strengthen sports personnel management and sports system perfection, and then improved Guangzhou city sports public service into higher levels [3]. $\mathrm{Li} \mathrm{Xi}$-Ming in the article "Chinese sports public service equalization's fiscal guarantee", by researching on Chinese sports public service equalization phenomenon, analyzed its status, and then further researched its fiscal guarantee mechanism. The thesis pointed out that with regard to other world sports power, China hasn't yet done a good job in sports public service, fiscal guarantee mechanism hasn't yet so perfected, it should take world main countries as examples in the future to propel to Chinese sports public service undertaking development [4].

The paper researches on Chinese rural sports public service funds input and current status, by analyzing its historical data, it predicts on Chinese rural sports public service funds input tendency in future five years, and further analyzes its causes to provide theoretical basis for Chinese rural sports public service development.

\section{TREND EXTRAPOLATION MODEL-BASED SPORTS FUNDS INPUT PREDICTION MODEL ES- TABLISHMENT}

Rural sports is an important part in Chinese sports, its development is related to Chinese sports overall advancement. In current stage, Chinese rural sports has many problems, inputs shortage, structural imperfection, sports consciousness not going deeper and others, all of them are factors that restrict its development. 


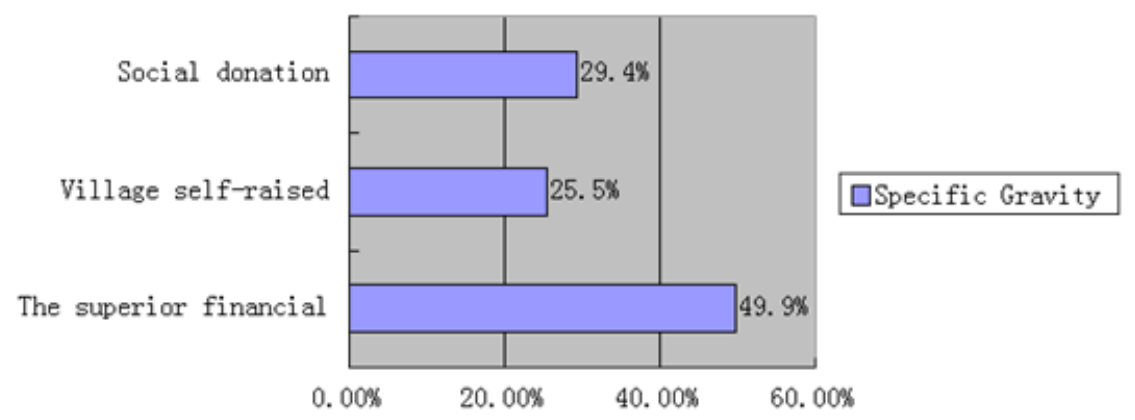

Fig. (1). Sports funding sources.

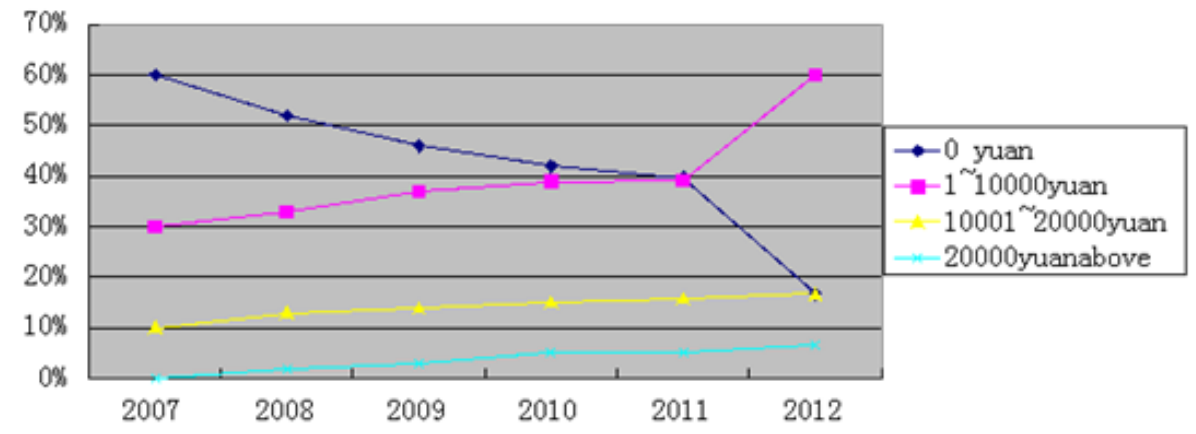

Fig. (2). Our country rural sports expenditure percentage forecast.

Table 1. 2007 2012 Chinese rural sports funds input situation.

\begin{tabular}{|c|c|c|c|c|}
\hline Sports Funds (\%) & 0Yuan & $\mathbf{1}$ 10000Yuan & 10001 20000Yuan & Above20000Yuan \\
\hline \hline 2007 & $60 \%$ & $30 \%$ & $10 \%$ & $2 \%$ \\
\hline 2008 & $52 \%$ & $33 \%$ & $13 \%$ & $3 \%$ \\
\hline 2009 & $46 \%$ & $37 \%$ & $15 \%$ & $5 \%$ \\
\hline 2010 & $42 \%$ & $39 \%$ & $15.8 \%$ & $5.3 \%$ \\
\hline 2011 & $39.7 \%$ & $39.2 \%$ & $16.7 \%$ & $6.6 \%$ \\
\hline 2012 & $16.7 \%$ & $60 \%$ & & \\
\hline
\end{tabular}

From above Fig. (1) Chinese rural sports funds input sources, it is clear that Chinese rural sports funds source is single, superior finance is the main factor to support its development, next is social donation, village self-raised sports funds are few, which indicates Chinese rural sports is confronting drawbacks of insufficient input in the field of input.

By far, Chinese rural sports development suffers rural economic influence, is far away from city sports. Rural sports all aspects' construction has not yet been perfected and its funds input is also inferior to city sports. Below Table $\mathbf{1}$ is Chinese rural sports' funds input sum percentage of total fiscal expenditure during 2007 to 2012, data is from Chinese statistical yearbook.

For above table data, it carries out trend extrapolation prediction analysis, and then predicts changing situation of Chinese rural sports funds input sum percentage of total fiscal expenditure as Fig. (2).

\subsection{Trend Extrapolation Prediction Approach Guiding Thought}

Trend extrapolation predication method is one of methods in time sequence, its main thought is finding out things development general rules according to their development history and reality information, and further predicating development trend in some time in future based on this. Its predication method steps are mainly following Fig. (3).

Trend extrapolation prediction method common used mathematical models are exponential curve approach, modified exponential curve approach, logistic curve (growth curve), envelop curve and so on. For Chinese rural sports public service funds input prediction, it belongs to trend extrapolation prediction approach researching scope, here applies modified exponential curve approach and logistic curve to predict on Chinese rural sports funds input. 


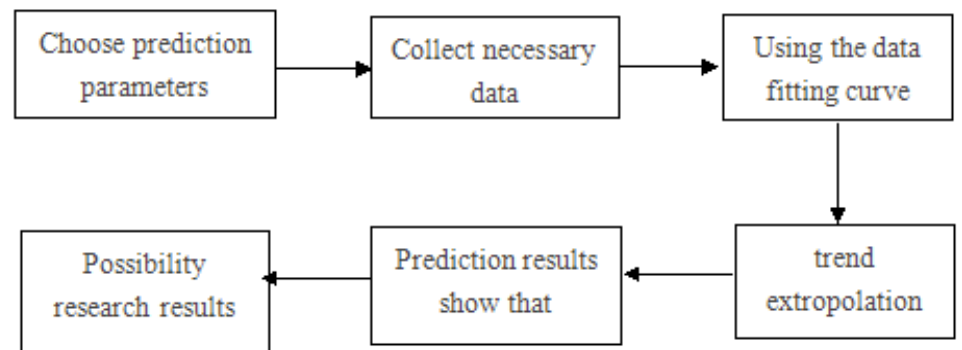

Fig. (3). Trend extrapolation prediction steps.

\subsection{Utilize Modified Exponential Curve Approach to Predict Chinese Rural Sports Funds Input Situation}

All things development has certain limitations, modified exponential curve overcomes exponential curve method drawback of predicted value being infinitely increased with time passing when predict, its prediction gets closer to actual things change rules. Modified exponential curve method mathematical model is:

$\hat{y}_{t}=K+a b^{t}$

Among them, $K, a, b$ should be defined with historical data.

For following phenomena:

In the preliminary stage, growth is rapid, and subsequently growth rate gradually reduces ;

When $K>0, a<0,0<b<1, \quad t \rightarrow \infty, a b^{t} \rightarrow 0$, that $\hat{y}_{t} \rightarrow K$

When $K$ value can be implemented and defined, adopt least square method to define parameters in model, and when $K$ value cannot be defined in advance, adopt three sums method.

Divide time sequence corresponding $n$ pieces of observation value into three parts, and three parts are equal as well as every part totally has $m$ periods that is also $n=3 m$.

Part one: $y_{1}, y_{2}, y_{3}, \cdots, y_{m}$

Part two: $y_{m+1}, y_{m+2}, y_{m+3}, \cdots, y_{2 m}$;

Part three: $y_{2 m+1}, y_{2 m+2}, y_{2 m+3}, \cdots, y_{3 m}$

Among them, every part trend sum is equal to corresponding observation value sum, and therefore, it provides parameters estimation, three sums method steps are as following:

Record observation value each part sum is:

$S_{1}=\sum_{t=1}^{m} y_{t}, S_{2}=\sum_{t=m+1}^{2 m} y_{t}, S_{3}=\sum_{t=2 m+1}^{3 m} y_{t}$,

And it has:

$$
\left\{\begin{array}{l}
S_{1}=\sum_{t=1}^{m} \hat{y}_{t}=\sum_{t=1}^{m}\left(K+a b^{t}\right)=m K+a b\left(1+b+b^{2}+\cdots+b^{m-1}\right) \\
S_{2}=\sum_{t=m+1}^{2 m} \hat{y}_{t}=\sum_{t=m+1}^{2 m}\left(K+a b^{t}\right)=m K+a b^{m+1}\left(1+b+b^{2}+\cdots+b^{m-1}\right) \\
S_{3}=\sum_{t=2 m+1}^{3 m} \hat{y}_{t}=\sum_{t=2 m+1}^{3 m}\left(K+a b^{t}\right)=m K+a b^{2 m+1}\left(1+b+b^{2}+\cdots+b^{m-1}\right)
\end{array}\right.
$$

Among them: $\left(1+b+b^{2}+\cdots+b^{m-1}\right)(b-1)=b^{m}-1$

It further can get:

$\left\{\begin{array}{l}S_{1}=m K+a b \frac{b^{m-1}}{b-1} \\ S_{2}=m K+a b^{m+1} \frac{b^{m-1}}{b-1} \\ S_{3}=m K+a b^{2 m+1} \frac{b^{m-1}}{b-1}\end{array}\right.$

Thereupon, it can get:

$\left\{\begin{array}{l}b=\left(\frac{S_{3}-S_{2}}{S_{2}-S_{1}}\right)^{\frac{1}{m}} \\ a=\left(S_{2}-S_{1}\right) \frac{b-1}{b\left(b^{m}-1\right)^{2}} \\ K=\frac{1}{m}\left[S_{1}-\frac{a b\left(b^{m}-1\right)}{(b-1)}\right]\end{array}\right.$

Besides, when predict data, it should test data, test method is:

$\frac{y_{t+1}-y_{t}}{y_{t}-y_{t-1}} \approx b$

\subsection{Modified Exponential Curve Approach Data Han- dling and Relevant Conclusion}

For Table 1 data, utilize above guiding thoughts to analyze, now divide table's 6 data into three parts, every part has 2 data, that $n=6, m=2$, and regard year 2007 as its starting year that $t=1$.

Predict on percentage that sports fund is 0 Yuan

Firstly, by calculation, it easily gets: $\frac{y_{t+1}-y_{t}}{y_{t}-y_{t-1}} \in[0.55,1.25]$ 
According to formula(2)it gets: $S_{1}=18.72, S_{2}=$ $19.52, S_{3}=19.93$

Then by formula(5)it gets: $b=0.8003, a=-84.022, K=$ 6.78708

So obtained Chinese rural sports funds input percentage modified exponential curve mathematical model is:

$$
y=K+a b^{t}=6.78708-84.022 \times 0.8003^{t}
$$

Predict on percentage that sports fund is $1 \sim 10000$ Yuan

Firstly, by calculation, it easily gets: $\frac{y_{t+1}-y_{t}}{y_{t}-y_{t-1}}$ $-\in[0.51,1.05]$

According to formula(2)it gets: $S_{1}=18.65, S_{2}=$ $19.43, S_{3}=19.88$

Then by formula(5)it gets: $b=0.8012, a=-84.011$, $K=6.77541$

So obtained Chinese rural sports funds input percentage modified exponential curve mathematical model is:

$$
y=K+a b^{t}=6.7541-84.011 \times 0.8012^{t}
$$
Yuan

Predict on percentage that sports fund is 10001 20000

Firstly, by calculation, it easily gets: $\frac{y_{t+1}-y_{t}}{y_{t}-y_{t-1}} \in$

$[0.46,1.11]$
According to formula(2)it gets: $S_{1}=17.89, S_{2}=18.41$, $S_{3}=19.75$

Then by formula(5)it gets: $b=0.8001, a=-81.025$, $K=6.5468$

So obtained Chinese rural sports funds input percentage modified exponential curve mathematical model is:

$$
y=K+a b^{t}=6.5468-81.025 \times 0.8001^{t}
$$
Yuan

Predict on percentage that sports fund is above 20000

Firstly, by calculation, it easily gets: $\frac{y_{t+1}-y_{t}}{y_{t}-y_{t-1}}$

$\in[0.37,0.98]$

According to formula(2)it gets: $S_{1}=16.75, S_{2}=17.68$, $S_{3}=18,91$

Then by formula(5)it gets: $b=0.7901, a=-80.0301$, $K=6.0031$

So obtained Chinese rural sports funds input percentage modified exponential curve mathematical model is:

$$
y=K+a b^{t}=6.0031-80.0301 \times 0.7901^{t}
$$

When predict Chinese rural sports funds input development change in future five years after 2012, only need to input $t$ value into above formula, as predicting

\begin{tabular}{|c|c|c|c|c|c|}
\hline Sports funds & 2013 & 2014 & 2015 & 2016 & 2017 \\
\hline 0Yuan & $15.4 \%$ & $15.0 \%$ & $14.6 \%$ & $14.1 \%$ & $13.2 \%$ \\
\hline $1 \sim 10000$ Yuan & $61.5 \%$ & $61.7 \%$ & $62.9 \%$ & $63.0 \%$ & $63.4 \%$ \\
\hline 10001 20000Yuan & $17.2 \%$ & $17.9 \%$ & $18.0 \%$ & $19.5 \%$ & $20.1 \%$ \\
\hline Above 20000 Yuan & $7.0 \%$ & $7.4 \%$ & $7.8 \%$ & $8.0 \%$ & $8.3 \%$ \\
\hline
\end{tabular}
$y_{2013}$, then it has $t=2013-2004+1=10$. It further gets following Table 2 prediction result:

Table 2. Chinese rural sports funds input percentage prediction.

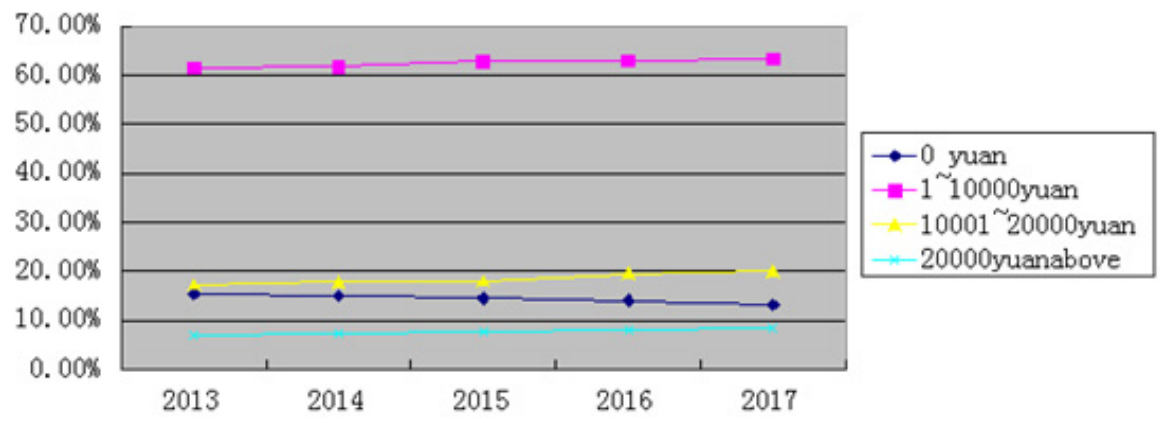

Fig. (4). Our country rural sports expenditure percentage forecast. 
Table 3. Rural residents' sports consciousness statistics.

\begin{tabular}{|c|c|c|c|c|}
\hline & More Necessary & Necessary & No Need & Incomprehension \\
\hline \hline Proportion $\%$ & $15.4 \%$ & $47.7 \%$ & $17.5 \%$ & $19.3 \%$ \\
\hline
\end{tabular}

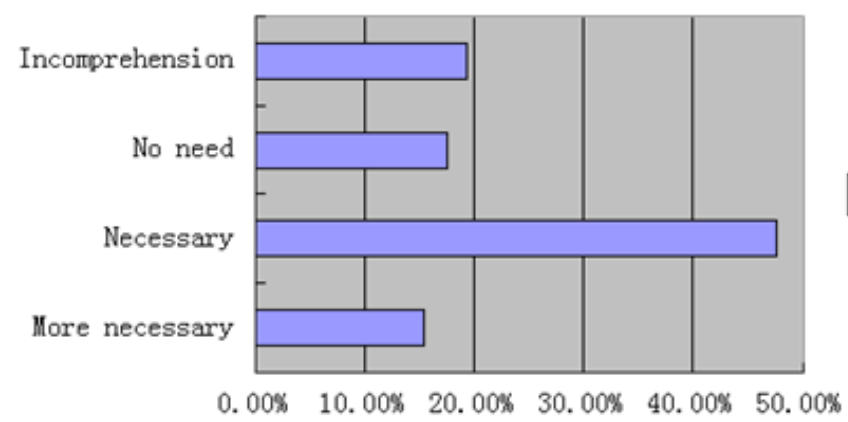

Fig. (5). Rural residents' sense of sports statistics.

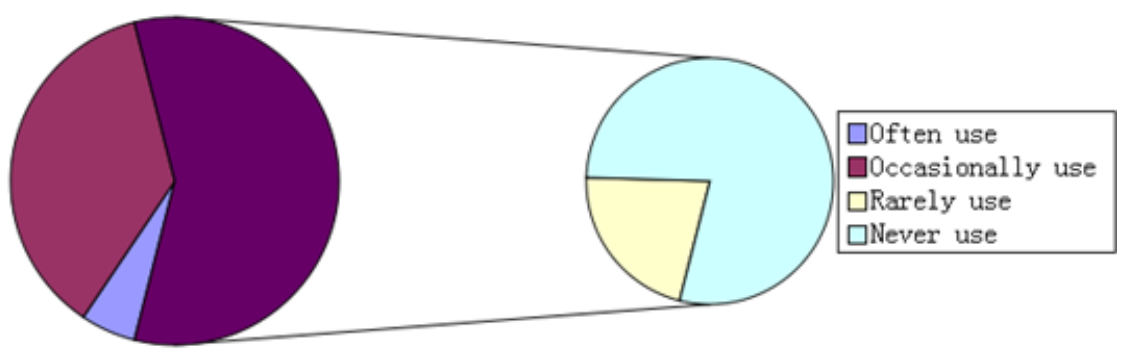

Fig. (6). Rural sports facilities utilization statistics.

Compare with Figs. (2 and 4) data statistical figure, it can get conclusion: no matter historical data of Chinese rural sports funds input occupied percentage of total fiscal expenditure during 2007 to 2012, or predicted data during 2013 to 2017, the sizes are constantly growing, but amplification is not big, change is not obvious. Though rural sports funds input's zero input proportion is decreasing, but decreasing rate is smaller. From historical data figure and prediction figure comparative analysis, Chinese rural sports funds input is basically around 10000 Yuan, it will not significant change in recent five years [5].

\section{CHINESE RURAL SPORTS PUBLIC SERVICE CONFRONTED CURRENT SITUATION}

Sports consciousness is foundation of sports development and also its essence, strong sports consciousness is helpful for conducting sports activities in masses life and propelling to sports public service transmission. Below Table $\mathbf{3}$ is Chinese rural residents' sports consciousness extents statistics, make statistical analysis of it and get conclusions.

By statistical analysis Fig. (5), it can get conclusion that Chinese rural residents' sports consciousness is better, $47.7 \%$ groups think proceed with sports activities is more necessary. But there are still $17.5 \%$ groups think that sports activities is not necessary, and $19.3 \%$ groups haven't yet comprehended on sports activities importance. It restricts Chinese rural sports public service transmission and development among masses life to a certain extent.
Sports facilities soundness is the foundation of spreading sports in villages. Good sports facilities can arouse masses positivity and enthusiasm in participating in sports exercise, and then more eagerly participate in rural sports activities, and meanwhile also propel to sports transmission among residents' life.

Above statistical Table $\mathbf{4}$ and Fig. (6) analysis shows that though China's many villages have set up sports activity facilities, due to rural residents' conditions' limitation and sports consciousness and other factors, the utilization rate is not high, $50.0 \%$ sports facilities are basically not used, often used sports facilities only are $6.0 \%$. It shows Chinese rural sports facilities utilization situation is not going well, many sports facilities become decorations.

Except for sports facilities construction, sports field condition is also another important factor that restricts Chinese rural sports public service development. In village, it mainly has stadium, body-building plaza, non-standard sports ground and basketball court, tourism and leisure post and others multiple sports activity places to masses to select.

By above Table 5 and Fig. (7) analysis, it can get conclusion that among sports grounds construction, village per capita owned area is little. Rural stadium, tourism and leisure post don't exist at all, body-building plaza's per capita owned area is $28.94 \%$, non-standard sports ground's per capita owned area is $27.99 \%$, basketball court's per capita owned area is $43.07 \%$. It is clear that village sports activity field construction hasn't yet been perfected. 
Table 4. Rural sports facilities utilization situation.

\begin{tabular}{|c|c|c|c|c|}
\hline & Often Use & Occasionally Use & Rarely Use & Never Use \\
\hline \hline Proportion\% & $6.0 \%$ & $40.3 \%$ & $13.7 \%$ & $50.0 \%$ \\
\hline
\end{tabular}

Table 5. Villages all kinds of sports activity places percentages of total places.

\begin{tabular}{|c|c|c|c|}
\hline Type of Sports Ground & Number of Grounds (\%) & Gross area (\%) & Per Capita Owned Area (\%) \\
\hline \hline Stadium & $0 \%$ & $0 \%$ & $0 \%$ \\
\hline Body-building plaza & $25.93 \%$ & $49.89 \%$ & $28.94 \%$ \\
\hline Non standard sports ground & $18.52 \%$ & $30.10 \%$ & $27.99 \%$ \\
\hline Basketball court & $55.55 \%$ & $30.01 \%$ & $43.07 \%$ \\
\hline Tourism and leisure post & $0 \%$ & $0 \%$ & $0 \%$ \\
\hline
\end{tabular}

Table 6. Rural sports personnel allocation situation.

\begin{tabular}{|c|c|c|c|c|c|c|c|}
\hline & \multicolumn{2}{|c|}{ Present Sports Instructors } & \multicolumn{2}{|c|}{ Present Activity Site } & \multicolumn{3}{|c|}{ Activity Way } \\
\hline & Have & Don't have & Have & Don't have & Basketball Game & Cultural Activities & Others \\
\hline Proportion\% & $16.7 \%$ & $83.3 \%$ & $23.3 \%$ & $76.7 \%$ & $38.4 \%$ & $50.0 \%$ & $11.6 \%$ \\
\hline
\end{tabular}

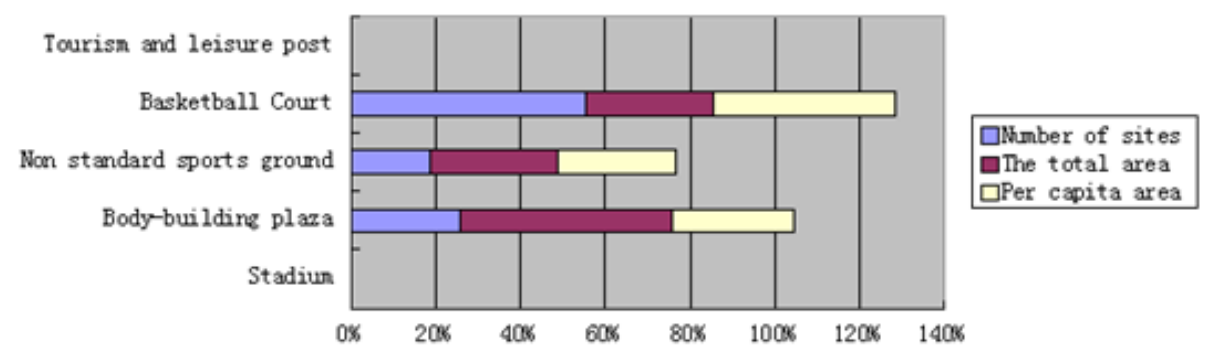

Fig. (7). Area percentage of total site country all kinds of sports activities.

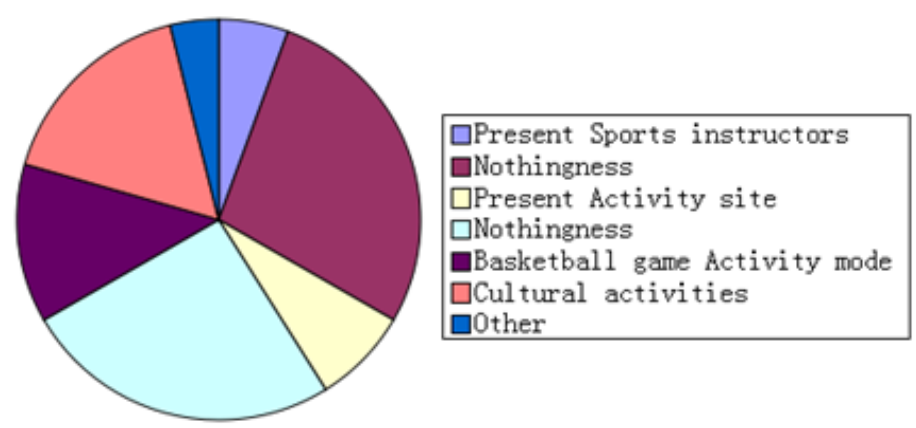

Fig. (8). Rural sports match situation.

Sports administrative staff is powerful guarantee for perfecting sports development and normalizing sports behaviors. Village sports personnel allocation is the foundation to propel to rural sports advancement and manage rural sports public service.

Above statistical Table 6 and Fig. (8) indicates that Chinese rural sports public service staff allocation is not very perfect, present sports instructors are only $16.7 \%$, present activity site only accounts for $23.3 \%$. Besides, basketball game, cultural activities are also fewer. It is another important cause that affects Chinese sports public service development.

Though it has relative sound sports facilities and sports grounds, and also relative better sports service staff allocation, equipment maintenance is still an important problem. Only maintain sports equipment and sports grounds well then can guarantee residents' sports activities smoothly proceeding. 


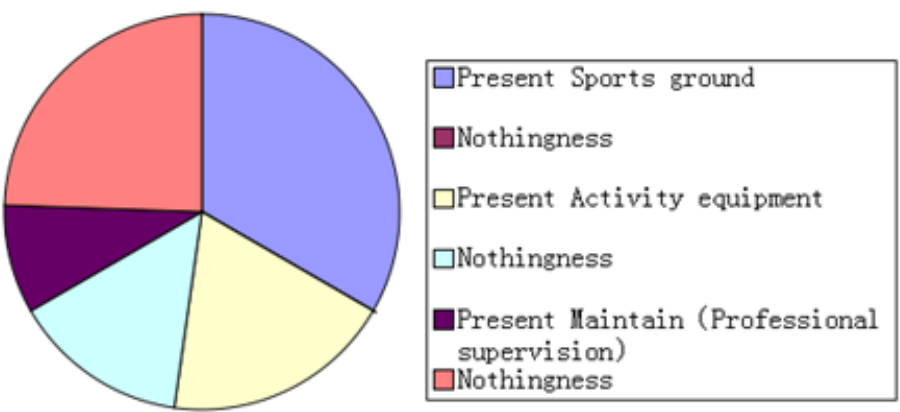

Fig. (9). Rural sports venues and equipment maintenance.

Table 7. Rural sports ground and equipment maintenance.

\begin{tabular}{|c|c|c|c|c|c|c|}
\hline \multirow{2}{*}{} & \multicolumn{2}{|c|}{ Present Sports Ground } & \multicolumn{2}{c|}{ Present Activity Equipment } & \multicolumn{2}{c|}{ Present Maintenance (Professional Supervision) } \\
\cline { 2 - 7 } & Have & Don't have & Have & Don't have & Have & Don't have \\
\hline \hline Proportion\% & $100.0 \%$ & $0 \%$ & $56.7 \%$ & $43.3 \%$ & $26.6 \%$ & $73.4 \%$ \\
\hline
\end{tabular}

From Table 7 and Fig. (9), it is clear that sports ground and sports equipment lack of necessary maintenance and professional supervision so that lead to equipment damage speed to accelerate and reduce its life time. Irregular sports ground and equipment maintenance way let rural sports ground and equipment utilization to be tighter to a certain extent.

\section{CONCLUSION}

The paper firstly establishes trend extrapolation prediction model to predict Chinese rural sports public service funds input. By analyzing Chinese rural sports funds input historical data, it applies modified exponential curve approach to make quantitative analysis of Chinese rural sports public service funds input during 2007 to 2012, get predicted mathematical model and then predicts its changes. Finally it gets conclusion that though Chinese rural sports funds input percentage of total fiscal expenditure is constantly increasing, amplification is not big, and so change is not obvious.

Secondly, it makes statistical analysis of Chinese rural sports public service confronted status, carries on specific research from sports consciousness, sports facilities utilization, sports activity ground construction, sports personnel management system, sports ground and equipment maintenance these five aspects, by statistical table and statistical figure, compares data change trends, and finally gets conclusion that Chinese rural sports residents' sports consciousness is not strong, sports facilities utilization is incomplete, sports activities ground construction is imperfect, sports personnel management system is unsound, sports ground and equipment maintenance is not formal, all of these are key problems that restrict Chinese rural sports public service development.

\section{CONFLICT OF INTEREST}

The authors confirm that this article content has no conflict of interest.

\section{ACKNOWLEDGEMENTS}

Declared none.

\section{REFERENCES}

[1] C. Yang, and M.A. Ge-sheng, "An empirical study on community sports service residents' satisfaction index model," China Sport. Sci. Technol., vol. 45, no. 4, 2009.

[2] H. Ying, and X. Ming, "Theoretical and empirical study on evaluation mode of sports service satisfaction degree in city community," J. Wuhan Inst. Phys. Educ., vol. 41, no. 11, pp. 40-42, 2007.

[3] L. Bao, H. Shan-lian, X. Hai-xia, and G. Jian-hui, "Indices of the equality of essential public health services in China," Chin. J. Health Pol., vol. 2, no. 6, pp. 13-17, 2009.

[4] Z. Da-chao, and L. Min, "Studies on evaluation index system of public sports facilities development level in China," Chin. Sport. Sci., vol. 33, no. 4, pp. 3-23, 2013.

[5] Z. Jie, and W. Ying, "The evaluation index system of extracurricular sports activities in secondary schools in shanghai under the background of "sunshine sports"," J. Shanghai Phys. Educ. Inst., no. 6 , pp. 80-82, 2012

(C) Cai and Wang; Licensee Bentham Open

This is an open access article licensed under the terms of the (https://creativecommons.org/licenses/by/4.0/legalcode), which permits unrestricted, noncommercial use, distribution and reproduction in any medium, provided the work is properly cited. 